\title{
Politicas públicas locais para promoçâo da atividade física das crianças nos parques públicos
}

| ${ }^{1}$ Simone Medeiros de Oliveira, ${ }^{2}$ Maria Paula Maia dos Santos, ${ }^{3}$ Paula Silva |

Resumo: Evidências revelam o potencial dos parques públicos para a promoção da atividade física e da saúde. A valorização desses ambientes para a promoçáo da atividade física das crianças pode ser uma mais-valia nas políticas públicas de promoção da saúde e prevenção do sedentarismo das crianças. $\mathrm{O}$ objetivo deste estudo foi a análise das políticas públicas de promoção da atividade física das crianças em parques públicos do Município de Guimarães, Portugal. Foram realizadas entrevistas semiestruturadas individuais a gestores políticos públicos locais. O tratamento dos dados foi realizado segundo a metodologia qualitativa de análise do conteúdo, donde emergiram os seguintes temas relacionados com a promoção da atividade física das crianças: parceria política (pública e intersetorial), as estratégias e os constrangimentos locais. Os resultados revelaram a necessidade de maiores esforços públicos para a valorização dos parques públicos para a promoção da atividade física das crianças. Promover políticas públicas locais que favoreçam a parceria intersetorial local poderá ser uma estratégia eficaz para a promoção da atividade física das crianças nos parques públicos.

> Palavras-chave: política pública; parques recreativos; criança; exercício.

\author{
${ }^{1}$ Faculdade de Desporto, \\ Universidade do Porto. Porto, \\ Portugal (simonemedeiros2005@ \\ hotmail.com). \\ ORCID: 0000-0002-3700-4887 \\ 2 Faculdade de Desporto, \\ Universidade do Porto. Porto, \\ Portugal (msantos@fadeup.pt). \\ ORCID: 0000-0002-2182-9841 \\ ${ }^{3}$ Faculdade de Desporto, \\ Universidade do Porto. Porto, \\ Portugal (psilva@fadeup.pt). \\ ORCID: 0000-0003-4231-9469
}

Recebido em: 28/05/2019 Aprovado em: 22/05/2020 Revisado em: 24/03/2021 


\section{Introdução}

Com o aumento da urbanização, o dominante ambiente construído das cidades emerge na literatura como um fator determinante das oportunidades de prática da atividade física (AF) (SALLIS et al., 2012). Por sua vez, estudos no âmbito da AF e saúde dos indivíduos alertam para as condicionantes dos meios urbanos, especialmente devido ao elevado índice de sedentarismo (KOHL et al., 2012).

A oferta de ambientes urbanos que proporcionem a oportunidade da prática da AF é uma tendência de destaque nas atuais políticas públicas, com o crescente investimento em Espaços Verdes, ambientes ao ar livre associados a melhorias na saúde dos indivíduos (LEE et al., 2015), sendo que sua principal função pode estar associada ao aumento do bem-estar de seus utilizadores (ADINOLFI et al., 2014). Os parques públicos (PP) são modelos comuns de Espaços Verdes urbanos associados à prática da AF (KACZYNSKI; HENDERSON, 2008). A presença de estruturas que facilitam o envolvimento em práticas fisicamente ativas, em especial a AF recreativa (TESTER, 2009), é uma característica que pode favorecer a utilização dos PP pelas crianças para a prática da AF.

Em crianças, verifica-se a associaçáo positiva entre a exposição aos Espaços Verdes e seu bem-estar emocional (WARD et al., 2016), sendo que uma maior frequência a estes ambientes tem efeito positivo sobre a qualidade de vida das mesmas (McCRACKEN et al., 2016). Estudos indicam que em ambientes ao ar livre, as crianças são mais ativas na presença de seus pares (DUNTON et al., 2011; PEARCE et al., 2014), pelo que, estratégias de maiores oportunidades de utilização dos PP pelas crianças são necessárias nas políticas públicas de promoção da AF.

Segundo o modelo proposto por Bedimo-Rung et al. (2005), sobre os benefícios proporcionados aos indivíduos pela prática da $\mathrm{AF}$ nos $\mathrm{PP}$, a utilização destes ambientes para a prática da AF pode ser suportada por políticas relacionadas com a gestão e com o orçamento local. A compreensão das políticas locais de incentivo à $\mathrm{AF}$ das crianças nos $\mathrm{PP}$ permite a caracterização dos potenciais atributos destes ambientes locais para a promoção da saúde das crianças, como também, a elaboração de estratégias eficazes de combate ao sedentarismo.

Em Portugal, a promoção do estilo de vida ativo é estimulada através do Programa Nacional para a Promoção da AF (DIREÇÃO-GERAL..., 2017), sendo o fomento de ambientes ao ar livre facilitadores da prática da AF uma das estratégias 
norteadoras. O Município de Guimarães, localizado na região norte de Portugal, é reconhecido pelo incentivo à prática de $\mathrm{AF}$, tendo sido a "Capital Européia do Desporto” em 2012. Atualmente, disponibiliza à população local uma ampla diversidade de estruturas desportivas e recreativas (CÂMARA..., 2017a), onde a valorização das áreas verdes é evidenciada pela distribuição de 24 Espaços Verdes ao longo do seu território (CÂMARA..., 2017b).

$\mathrm{O}$ incentivo à prática da $\mathrm{AF}$ ao ar livre integra uma estratégia prioritária de Saúde Pública em Portugal (DIREÇÃO-GERAL..., 2017), cenário ainda pouco estudado para a população das crianças. Conhecer as políticas públicas locais de promoção da AF das crianças nos PP de um município que investe nesta área, a exemplo de Guimarães, pode evidenciar o potencial destas práticas públicas para a promoção da AF ao ar livre e seus benefícios à saúde das crianças. Assim, o objetivo deste estudo foi a análise das políticas públicas de promoção da AF das crianças em PP no Município de Guimarães.

\section{Metodologia}

Este estudo qualitativo foi aprovado pelo Comitê de Ética da Faculdade do Desporto da Universidade do Porto, Portugal (processo CEFADE 07.2015) e seus resultados integram uma tese de doutorado em Atividade Física e Saúde.

Visando à obtenção de dados que revelem os significados, as relaçóes e os processos (BARDIN, 2008) presentes nas políticas públicas locais de promoçáo da AF das crianças em PP, foram realizadas entrevistas semiestruturadas individuais a agentes públicos do Município de Guimarães: dois representantes da Câmara Municipal de Guimarães, responsáveis pelos setores do Desporto e da Educação, e quatro presidentes de Juntas de Freguesia do município, cujas localidades têm PP em áreas residenciais urbanas. $\mathrm{O}$ guia das entrevistas foi elaborado com base na revisão da literatura sobre o tema do estudo.

Os participantes foram previamente informados do objetivo e metodologia deste estudo, do sigilo e anonimato; tendo sido obtido o termo de consentimento livre informado de cada. As entrevistas, que decorreram nos gabinetes dos participantes em horário disponibilizado pelos mesmos, tiveram duração de aproximadamente 40 minutos com saturaçáo do tema. Os dados foram gravados em registro áudio e integralmente transcritos, assinalando as pausas e as interjeições. 
Para análise dos dados, optou-se pelo tema como unidade de registro na análise do conteúdo, sendo que os dados foram sujeitos a uma análise indutiva com o recurso ao programa QSR NVivo 11 para a sua gestão e codificação. O processo de análise do conteúdo foi realizado de forma independente por duas das autoras deste estudo, tendo uma a responsabilidade da edição do processo de codificação. Os resultados foram comparados e discutidos até ser obtido um consenso. Estes procedimentos de análise e discussão foram acompanhados por uma terceira autora no sentido de promover o confronto de crenças, valores e preconceitos.

\section{Análise e Discussão dos Resultados}

A análise dos resultados realçou quatro temas importantes para a compreensão das políticas públicas locais de promoção da AF das crianças, em especial nos PP, que a seguir são apresentados e discutidos concomitantemente.

\section{Parceria pública local}

$\mathrm{Na}$ análise dos dados, a parceria pública local destacou-se nos relatos dos entrevistados, evidenciando relaçôes entre os dois níveis (municipal e local) de agentes públicos entrevistados, que podem favorecer a promoção da AF das crianças nos PP locais.

A parceria entre os entrevistados foi evidenciada nos relatos através de açóes locais de manutenção e renovação dos PP, conforme verifica-se nos seguintes relatos:

No ano passado já fizemos algumas coisas, não temos orçamento, mas fizemos com a ajuda do Município [...] (E4).

A gestão do imobiliário, quem o instala é a Câmara, toda a manutenção, quem faz a manutençấo e limpeza de todo o espaço [...] (E3).

A existência de relaçóes de apoio entre os agentes públicos locais para a manutenção e a renovação dos PP revela-se como uma importante parceria que pode favorecer a promoção da AF das crianças nos PP locais, visto que a renovação de PP está associada a um maior número de crianças utilizadoras dos parques (COHEN et al., 2015). Contexto este, que parece favorecer a adoção do comportamento ativo pelas crianças em ambientes ao ar livre.

Kruger et al. (2010), ao analisarem as leis estatuais norte-americanas de suporte aos $\mathrm{PP}$, verificaram a prevalência de leis relacionadas à preservação e à conservação 
destes ambientes, destacando assim, a relevância destas açôes para a promoção da utilização dos PP. Fato que traz à tona, a necessidade de parcerias firmadas através de acordos e leis locais, proporcionando condições de maior coesão política pública local; que podem favorecer a oferta nos PP e contribuir para a promoção da AF das crianças nestes ambientes.

No Município de Guimarães, esta coesão política se destaca nos relatos dos entrevistados com o fomento de novos ambientes de promoção do lazer e da prática da AF:

Como a Câmara, entretanto, decide alargar aquele terreno que até então era estaleiro municipal, entendemos nós, junto em Câmara, como é lógico uma parceria, o fato de irmos fazer uma obra nos campos de futebol, que aquele terreno ficasse naturalmente bem com uma zona envolvente de lazer. (E5)

E então, como à partida o Município já se, não digo comprometeu, mas pronto, à partida vai haver ali obras [...] depois, a partir do momento em que podemos ter mais um espaço para rentabilizar para o desporto. (E4)

Em Portugal, dotar as localidades com ambientes que facilitem a prática de AF é uma estratégia prioritária da política nacional de Saúde (DIREÇÃO-GERAL..., 2017). Condição assegurada no Município de Guimarães pela parceria pública local, identificada nos relatos dos agentes públicos entrevistados. Desta maneira, certificase que o suporte de estratégias nacionais pode refletir em melhores condições de diálogo e interação entre os agentes públicos locais, fortalecendo e estabelecendo parcerias públicas para benefício da comunidade local.

A presença de PP e a parceria entreos agentes públicos locais são condiçôes favoráveis para a promoção da AF local. Os resultados revelaram que açóes de manutenção e a renovação dos PP locais podem ser beneficiadas com o estabelecimento da parceria pública local. De maneira que, a existência de uma relação favorável entre os agentes públicos, assegurada por acordos e leis, pode ser uma via estratégica de sucesso para promoção da AF das crianças nos PP locais.

\section{Parceria intersetorial local}

A cooperação de diversos setores no desenvolvimento de estratégias de promoção da saúde é uma tendência atual de políticas de Saúde Pública. Os benefícios desta parceria refletem-se numa melhor serventia pública à comunidade. No Município de Guimarães, a partir da análise das entrevistas realizadas, verifica-se que esta intenção 
está presente no discurso dos entrevistados, mas que ainda se revela distante da prática, surgindo nos relatos de forma segregada e não integrada, como pretendido:

[...] o que se nota é que as políticas de promoção do desporto e da atividade física e da qualidade de vida neste país, são políticas, são políticas individualizadas, de costas voltadas, quer dizer a Saúde não fala com o Desporto, o Desporto não fala com a Educação e a Educação não fala com a Saúde. (E1)

A ausência da partilha intersetorial é referida na literatura (LIBRETT et al., 2007) como uma barreira para a promoção da AF nos PP, caracterizando o desperdício da oportunidade de açôes eficientes de promoção da saúde dos indivíduos através da prática AF. A conversáo desta realidade, visando o envolvimento e o compromisso intersetorial local, poderá assegurar a desejada efetividade dessas estratégias de Saúde Pública.

As estratégias de promoção da AF em PP transpassam o domínio do setor público, abrangendo diversos setores que têm forte influência na dinâmica da comunidade local. Nesta realidade, a congregação intersetorial local estabelecida por meio de parcerias caracteriza uma via estratégica de sucesso para a implementação de açôes públicas de promoção da $\mathrm{AF}$ das crianças nos $\mathrm{PP}$ locais.

Projetos municipais que englobem a integralidade e a diversidade local podem criar as condiçôes favoráveis às parcerias intersetoriais com o poder público local. A exemplo do que foi identificado no Município de Guimarães, com a campanha municipal de candidatura à "Capital Verde Européia 2020”:

Portanto a questão da Capital Verde acabou por ser agregadora. É muito interessante a questâo, essa questão da Capital Verde e tem sido muito importante para o trabalho da própria autarquia. (E2)

Segundo a literatura, alguns temas reúnem características que podem proporcionar condiçôes favoráveis ao estabelecimento das desejáveis parcerias intersetoriais locais. Segundo Karjalainen et al. (2017), projetos sobre os determinantes ambientais de saúde podem contribuir para o estabelecimento das práticas políticas intersetoriais, pelo seu caráter multidisciplinar. Algumas características podem também agregar um elevado potencial de investimento público, conforme verifica-se ao nível europeu, onde verifica-se maior financiamento público de projetos sobre as determinantes ambientais do estilo de vida que integrem a AF, a nutrição e o comportamento (KARJALAINEN et al., 2017). De maneira que, a elaboração de estratégias públicas locais baseadas nestas determinantes ambientais de saúde poderá atrair e estimular a participação intersetorial local. 
Um estudo sobre práticas intersetoriais nas políticas da promoção da saúde dos indivíduos (AZEVEDO et al., 2012), realça o processo estratégico que envolve a ação prática de diferentes setores e não meramente a contribuição de seus conhecimentos. O que parece ser um desafio para a implementação de políticas públicas locais de promoção saúde das crianças através da AF nos PP do Município de Guimarães, visto a centralização das decisóes políticas referentes às açóes sobre a saúde, conforme verifica-se no relato a seguir:

Nós não temos as competências da Saúde, são muito centralizadas, portanto, fazem muito parte do governo central. (E2)

A vigilância cooperativa (elaboração, execução e acompanhamento intersetorial) das práticas de promoção da AF nos PP (KRUGER et al., 2007) pode ser uma via eficiente que promova o envolvimento e o comprometimento intersetorial nas ações de promoção da AF das crianças nos PP locais. O uso combinado (JACOBS, 2011) dos PP indica uma promissora estratégia de oportunidade da prática da vigilância cooperativa dos diversos setores locais. Os resultados do presente estudo revelaram o potencial do uso combinado dos PP para a integração dos diversos setores locais nas açôes de promoção da AF:

Ali no campo da feira, há um ginásio que aparece ali todos os domingos de manhã para fazer com que as pessoas apareçam e consigam então fazer de fato a atividade física. De fato, pensamos que só assim conseguimos motivar as pessoas [...] (E6).

Realidade identificada, mas que carece de políticas que sustentem este envolvimento e comprometimento intersetorial, uma vez que os resultados apontam para uma parceria pontual e esporádica. No Município de Guimaráes, identifica-se uma reduzida parceria entre o setor público e os demais setores locais, agravando o escasso cenário da promoção da AF nos PP locais:

É um assunto que várias vezes tem sido equacionado por nós, já fizemos uma e outra tentativa juntamente com alguns parceiros privados, no sentido de termos ao sábado de manhã digamos, alguém que possa apoiar (pausa) mas confesso que essas tentativas foram, não foram sucedidas [...] (E1).

Não resultou, os professores digamos assim, eram da “A.B”, das piscinas cá da freguesia. [...] os técnicos do desporto eram (pausa) e depois eles também têm que ver o que convém, o horário deles está todo preenchido, não é? Então eles tinham que fazer uma espécie de escala para ver quem é que estava naquele dia, quem é que não estava, para (pausa), pronto (E4).

Outra preocupante fragilidade identificada entre as parcerias intersetoriais locais neste estudo foi o distanciamento do setor comunitário nas estratégias públicas de 
promoção da AF local. A participação da comunidade nas políticas locais pode ser uma importante parceria intersetorial local para o sucesso das estratégias públicas de promoção da AF das crianças nos PP, visto que conforme a literatura, a parceria entre os órgãos legislativos locais e a comunidade pode fortalecer as estratégias de promoção da saúde das crianças (JUTTE et al., 2015). A presença de entidades ou organizaçóes comunitárias pode auxiliar a gestáo pública local, tornando assim, possível o engajamento comunitário nas políticas locais (MENDES et al., 2013). Maiores esforços devem ser feitos neste sentido, nomeadamente nas localidades estudadas, visando o estabelecimento desta parceria público-comunitária na formulação das políticas locais de promoção da saúde das crianças através da AF nos PP.

A parceria intersetorial tem um forte potencial sobre as políticas de promoção da AF (SPANGLER; CALDWELL, 2007), podendo revitalizar as atuais políticas locais de promoção da AF das crianças do Município de Guimarães. A criação de políticas públicas locais que fomentem a parceria intersetorial local, em especial os setores privados e comunitários, sugere ser uma via consistente para promoção da AF crianças nos PP das localidades estudadas.

\section{Estratégias locais de promoção da AF das crianças}

$\mathrm{O}$ universo das estratégias de promoção da AF é diversificado e complexo. $\mathrm{O}$ sucesso de uma estratégia eficaz de promoção da AF traduz-se pela definição de uma vertente de ação explícita, evitando o desgaste político e financeiro. No Município de Guimarães, esta definição está bastante clarificada nas estratégias de promoção da AF central com a oferta de ambientes que proporcionem a maior oportunidade da prática da AF ao ar livre, conforme verifica-se nos relatos dos entrevistados:

[...] temos investido muito na criaçáo de parques de lazer, urbanos, portanto, na Cidade e nas principais vilas, de forma que as pessoas possam utilizá-los enfim, nesta tal prática desportiva, portanto, informal e individual (E1).

[...] vamos ver se conseguimos ou não, porque o objetivo é mesmo este dotar a freguesia de mais Espaços Verdes. E de mais espaços para, para as pessoas fazerem desporto. (E4)

Todavia, este viés apresenta sua fragilidade. Conforme a literatura, a oferta de PP é uma estratégia comum de Saúde Pública, mas que não assegura a adoção do comportamento fisicamente ativo dos indivíduos utilizadores destes ambientes (AINSWORTH et al., 2007). Revelando o desafio enfrentado pela Saúde Pública 
na elaboração das estratégias de promoção da $\mathrm{AF}$ nos $\mathrm{PP}$ e a necessidade de açóes complementares à esta estratégia.

As estratégias de promoção da AF podem ser empregadas em diferentes contextos: recreativo, desportivo, escolar. A possibilidade de diversificação das estratégias nesses contextos é um elemento-chave para atingir a população das crianças. A oferta de estruturas desportivas é uma estratégia atraente às crianças, além de proporcionar condiçôes de uso diversificados dos PP e o pretendido envolvimento intersetorial local. Utilizá-la nas políticas públicas locais poderá ser via eficaz de promoção da AF das crianças. No Município de Guimarães esta é uma estratégia presente nas práticas públicas de promoção da $\mathrm{AF}$, referida pelos entrevistados:

Nós, tudo aquilo o que fazemos ao nível de desporto temos duas, duas vertentes: ou apoiar aqueles que de facto não praticam desporto ou apoiar grandes eventos desportivos para que captar gente para o desporto. (E1)

[...] portanto nós vamos relvar 2 campos de futebol, um que já existe de futebol 11, futebol sénior, sénior e camadas jovem, mas de futebol 11. E vamos construir um de 7, esse de raiz, que é paralelo [...] (E5).

O contexto escolar também se revela como um potente meio estratégico de promoção da AF das crianças. No Município de Guimarães, esta é uma prática presente nas políticas públicas de promoção da AF das crianças:

Nós temos, no âmbito da Educaçáo no $1^{\circ}$ ciclo, temos como atividade de enriquecimento auricular a atividade física desportiva [...] (E2)

Este ano estamos a lançar as bases de um outro programa muito interessante que é levar a atividade física regular aos jardins de infância. [...] (E1).

Dotar os ambientes de condiçóes adequadas, a exemplo da segurança (OLIVEIRA et al., 2018) e da qualidade dos PP; e de suporte poderá ser uma estratégia pública que promova a maior utilização dos PP pelas escolas para a promoção da AF das crianças. Contribuindo, para o combate ao sedentarismo e o alcance das recomendaçóes diárias da prática da AF das crianças (World Health Organization, 2010).

Outra intenção estratégica de promoção da AF das crianças no Município de Guimarães que merece destaque, é o propósito de "alcançar" as famílias através das crianças, com ações que possam contribuir para a adoção de hábitos saudáveis ao nível familiar:

Se levarmos o menino ao parque ele leva os pais. A nossa intervenção prioritária é efetivamente em contexto escolar. Levando os meninos a conhecer [estes espaços], eles levam os pais. (E2) 
Apesar da AF das crianças em ambientes ao ar livre ser potencializada pela presença de pares (DUNTON et al., 2011; BOCARRO et al., 2015), evidências revelam a importância da família para a adoção de comportamentos fisicamente ativos em crianças (JAGO et al., 2011; SANTOS et al., 2013; ROSS; FRANCIS, 2016). Assim, a ampliação das ações de promoção da utilização dos PP ao nível familiar é uma estratégia de vigor que deve ser disseminada nas políticas públicas de promoção da AF, uma vez que proporciona a oportunidade alargada da adoção do estilo de vida ativo; igualmente, os benefícios à saúde dos indivíduos do agregado familiar.

O potencial dos PP locais como ambiente estratégico de promoção da AF das crianças, parece estar subvalorizado no Município de Guimarães. Ao analisar as razões e os momentos em que os PP são promovidos pelos agentes públicos para a promoção da AF das crianças, fica evidente o papel secundário destes ambientes nas estratégias adotadas pelo poder público:

Nos outros meses quando estão conosco vão à uma visita no museu, vão, vão à um edifício
de interesse, e tem atividades até no Parque da Cidade [...] (E3).
[...] e nós mesmo no âmbito das escolas promovemos muito as atividades em que eles,
por exemplo, se vêm ao Castelo, se vão visitar um monumento, passem também por um
espaço verde, isto é, começa a fazer parte do roteiro, digamos assim, a valorização dos
espaços verdes. (E2)

Os PP têm uma elevada potencialidade de agregação da comunidade residente. A valorização destes ambientes através de estratégias públicas de promoção da AF das crianças em parceria com os diversos setores locais poderá beneficiar a comunidade promovendo a saúde, o bem-estar e a socialização local.

\section{Constrangimentos locais para a promoção da AF das crianças}

A implantação de estratégias nacionais de promoção da AF pode gerar condiçôes que sustentem as políticas de promoção da AF ao nível local. Em Portugal, uma das estratégias nacionais prioritárias da Direção Geral da Saúde é o Programa Nacional para a Promoção da AF (DIREÇÃO-GERAL..., 2017), sendo um reforço para as políticas locais de promoção da AF; reconhecido pelos entrevistados:

[...] e, portanto, também é difícil para uma Câmara Municipal tentar ela própria desenvolver um plano municipal, por exemplo, promoção do desporto, da saúde e do bem-estar, se depois não tiver meios financeiros ou se não, depois, não existir uma política que agregue isso tudo. (E1) 
Mas, uma assumida política nacional de promoção da AF não é a condição de garantia das práticas de promoção da AF local. A maior aproximação do poder público local às estratégias nacionais deve ser fomenta visando o empoderamento local das políticas nacionais existentes, ainda deficiente no Município de Guimarães, conforme revela o seguinte relato:

Portugal precisa urgentemente de um plano de promoção de, do desporto, do bem-estar e da qualidade de vida, ou seja, nada disso funcionará se nós não metermos na cabeça de que a promoçấo do desporto e da atividade física tem que ser um desígnio nacional. (E1)

Apesar dos entrevistados reconhecerem a importância das políticas nacionais para as práticas locais, verifica-se algum distanciamento entre os agentes públicos locais e as políticas nacionais. Maiores esforços devem ser feitos, por ambas as partes (local e nacional) para a extinção deste hiato, para benefício da comunidade local.

Para além das barreiras políticas, os resultados revelam outros constrangimentos que podem dificultar a promoção da AF das crianças nos PP locais. Apesar da oferta e a manutenção das estruturas dos PP integrarem as principais estratégias de promoção da $\mathrm{AF}$ local, estas também são relatadas como constrangimentos enfrentados pelos agentes públicos que podem afetar a promoção da AF das crianças nos PP, conforme verifica-se a seguir:

Há sempre problemas. Eu acho que, eu acho que necessariamente. Eu lembro-me que, por exemplo, faltavam os WC, umas casas de banho compatíveis com a qualidade que o parque oferecia. (E5)

Depois, aquela parte que tem as grades no, no campo? Aquilo precisa ali de uma remodelação. Precisa porquê? Pronto, na altura quando foi construído e pronto, muito bem, na altura entendeu-se, só que as coisas vão se degradando. Para além de se degradar, tem muitos buracos agora e há uma tendência a fugir as bolas, isso para além de, pronto ali temos... (E4).

A falta de recursos financeiros também representa alguns dos constrangimentos relatados pelos entrevistados na execução das estratégias de promoção da AF das crianças nos PP locais:

Pronto, os espaços em, com as condiçôes todas é uma prioridade, aquilo que nos dificulta mesmo é a questão do orçamento. (E4).

Com a disponibilidade de recursos humanos a ser afetada pelo orçamento financeiro do Município:

Mas, depois esta dificuldade, ou seja, nós temos algumas dezenas de parques de lazer espalhados por todo o território em Guimarães. E, portanto, isso teria que obrigar uma, a 
um esforço em termos de recursos humanos especializados, com um custo que nós neste momento não temos condições de suportar. (E1)

A limitação financeira representa uma importante barreira na implementação de estratégias de promoção da AF, já referida na literatura (LIBRETT et al., 2003), que pode afetar o cenário das práticas municipais de promoção da AF das crianças no PP. Todavia, as estratégias que envolvem a AF nos cuidados com a saúde dos indivíduos são provavelmente as açôes de promoção da Saúde Pública que envolvem menor investimento financeiro público, quando comparadas com outras estratégias comuns de cuidados na Atenção Primária à Saúde (VIJAY et al., 2015). Considerando que o planejamento distrital e local pode vir a contribuir na alocação dos recursos financeiros municipais para a promoção da AF (LIBRETT et al., 2003), a existência de projetos municipais de promoção da AF das crianças nos PP locais, sustentados por políticas nestes âmbitos, poderá garantir o financiamento e os recursos necessários para sua existência.

A promoção da AF das crianças nos PP locais também pode enfrentar outros constrangimentos que extrapolam as diretrizes políticas, infraestruturais e financeiras. No Município de Guimarães, o clima e suas adversidades foram referidos pelos entrevistados como barreiras para a promoção da $\mathrm{AF}$ das crianças ao ar livre, em especial nos PP:

Nós temos o inverno, geralmente chuvoso, não é? (pausa) E isto impede muito a utilização dos espaços verdes, dos espaços exteriores. (E2)

Os modelos sociais de conduta também foram mencionados como fatores limitadores da promoção da AF das crianças nestes ambientes, sendo que a conduta dos responsáveis de educação (em geral, os pais) das crianças em determinadas situações torna-se uma barreira para a prática de AF das crianças nos PP locais:

[...] não será fácil convencer os pais a deixarem os meninos irem à chuva, de bicicleta ou utilizar qualquer coisa nos espaços verdes. Portanto, nós temos muita dificuldade, penso que isso é uma questão de mentalidade, é uma questão que precisa ser trabalhada, mas os meninos têm que estar sempre muito salvaguardados, muito no seu espaço com todas as condiçóes e com, e com a temperatura regulamentar. [...] E, portanto, acham sempre que isso é perder tempo, o menino deve é estar na escola a aprender o português e a matemática e, portanto, como lhe digo assim, é uma questão muito transversal e muito de mudança de paradigmas. (E2). 
Interessantemente, alguns problemas sociais foram apontados pelos entrevistados como constrangimentos locais da promoçáo da AF das crianças nos PP, conforme os seguintes relatos:

Mas havia um grupo de jovens, pronto, aquela problemática, há quem diga que não, pronto, eu penso desta forma, mas nem toda a gente pensa como eu. Reuniam-se, pronto, dentro dessa problemática da droga, não é? (E4)

Por parte da população, não esquecer que nós temos uma população de baixas qualificações, e isto é sempre um problema porque são muito, é muito mais difícil convencer alguém com baixas qualificaçóes escolares do que com alta qualificaçâo escolar. (E2).

Trazendo à luz, o carácter multidisciplinar das estratégias de promoção da AF e a necessidade da prática integrada dos diversos setores e da comunidade local.

Numa perspectiva ecológica do comportamento da AF (SALLIS et al., 2006), onde se considera que esse comportamento sofra a influência de distintos níveis ambientais nos quais os indivíduos estão inseridos, a elaboração de políticas de promoção da AF das crianças deve prever condiçôes de enfrentamento dos diferentes constrangimentos locais. De forma que, políticas públicas locais que assegurem a efetividade das estratégias planejadas, e atenuem os principais constrangimentos locais existentes, podem contribuir para o sucesso da promoção da AF nos PP locais, em especial para a população das crianças.

\section{Conclusões}

Os resultados deste estudo revelam que a reduzida valorização dos PP locais para a prática de AF das crianças nas estratégias de política pública do Município de Guimarães. Onde, as estratégias públicas comuns de promoção da AF no Município de Guimarães, dinamizadas por meio da parceria pública local, estão relacionadas com a criação, a manutenção e a renovação dos PP. A implementação de estratégias específicas à população das crianças e a necessidade de políticas locais que promovam a parceria intersetorial na promoçáo da AF foram lacunas identificadas com a análise dos resultados sobre as políticas públicas de promoção da AF nos PP do Município de Guimarães.

A promoção da AF nos PP é uma estratégia de Saúde Pública de elevado potencial no enfrentamento do sedentarismo, em especial das crianças. A análise das políticas públicas vigentes e suas relaçôes contribui favoravelmente para a elaboração de estratégias de promoção da $\mathrm{AF}$ das crianças trazendo à tona a 
realidade local. A potencialização dos PP para a prática da AF das crianças, através de estratégias intersetoriais sustentadas por políticas públicas locais, é uma atual e importante determinante da AF que pode beneficiar a saúde dos indivíduos, em especial as crianças.

Este estudo revela resultados originais acerca de um tema carente na Saúde Pública que poderá incitar novas análises sobre este complexo tema. A reflexão sobre as políticas públicas locais para a promoção da AF das crianças nos PP proporciona proximidade com a realidade local, evidenciando as facilidades e as barreiras locais para a promoção da AF nos PP, em especial nos meios urbanos. A leitura do papel dos PP na Saúde Pública e sua dinâmica para a promoção da AF, em especial das crianças, constitui um novo horizonte de pesquisa que envolve um diversificado universo. Em futuros estudos pretende-se ampliar a análise dos demais agentes locais (pais, escolas, setor privado) potencializadores da promoçáo da AF das crianças nos PP locais. ${ }^{1}$

\section{Referências}

ADINOLFI, C.; SUÁREZ-CÁCERES, G. P.; CARIÑANOS, P. Relation between visitors' behaviour and characteristics of green spaces in the city of Granada, south-eastern Spain. Urban Forestry \& Urban Greening, v. 13, p. 534-542, 1/1/2014 2014.

AINSWORTH, B. E. et al. Perspectives of Public Health and Leisure Studies on Determinants of Physically Active Leisure. Journal of Physical Activity \& Health, v. 4, p. S24, 2007/04/02 2007. AZEVEDO, E. D.; PELICIONI, M. C. F.; WESTPHAL, M. F. Práticas intersetoriais nas políticas públicas de promoção de saúde. Physis Revista de Saúde Coletiva, v. 22, n. 4, p. 13331356, 2012.

BARDIN, L. Análise de Conteúdo. 4. Portugal: Ediçôes 70 Lda, 2008.

BEDIMO-RUNG, A. L.; MOWEN, A. J.; COHEN, D. A. The significance of parks to physical activity and public health: a conceptual model. Am J Prev Med, v. 28, n. 2 Suppl 2, p. 159-68, Feb 2005.

BOCARRO, J. N. et al. Social and Environmental Factors Related to Boys' and Girls' ParkBased Physical Activity. Prev Chronic Dis, v. 12, p. E97, 2015.

CÂMARA MUNiCIPAL DE GUIMARÃES. Cidade Desportiva. Câmara Municipal de Guimarães, 2017a. Disponível em: < http://www.cm-guimaraes.pt/pages/895 >. Acesso em: 10 jul. 2017.

. Espaços Verdes. Câmara Municipal de Guimarães, 2017b. Disponível em: < http:// www.cm-guimaraes.pt/pages/926?folders_list_29_page=2 >. Acesso em: 10 jul. 2017. 
COHEN, D. A. et al. Impact of park renovations on park use and park-based physical activity. J Phys Act Health, v. 12, n. 2, p. 289-95, Feb 2015.

DIREÇÃO-GERAL DA SAÚDE. Programa Nacional para a Promoção da Atividade Física. 2017. Disponível em: < https://www.dgs.pt/pns-e-programas/programas-de-saude-prioritarios/ atividade-fisica.aspx >. Acesso em: 5 mar. 2018.

DUNTON, G. F. et al. Physical and social contextual influences on children's leisure-time physical activity: an ecological momentary assessment study. J Phys Act Health, v. 8 supl 1, p. S103-8, Jan 2011.

JACOBS, J. Morte e vida de grandes cidades. São Paulo: WMF Martins Fontes, 2011.

JAGO, R. et al. Parenting styles, parenting practices, and physical activity in 10- to 11-year olds. Prev Med, v. 52, n. 1, p. 44-7, Jan 2011.

JUTTE, D. P.; MILLER, J. L.; ERICKSON, D. J. Neighborhood adversity, child health, and the role for community development. Pediatrics, v. 135 Suppl 2, p. S48-57, Mar 2015.

KACZYNSKI, A. T.; HENDERSON, K. A. Parks and recreation settings and active living: a review of associations with physical activity function and intensity. J Phys Act Health, v. 5, n. 4, p. 619-32, Jul 2008.

KARJALAINEN, T.; HOEVELER, A.; DRAGHIA-AKLI, R. European Union research in support of environment and health: Building scientific evidence base for policy. Environ Int, v. 103, p. 51-60, Jun 2017.

KOHL, H. W., 3rd et al. The pandemic of physical inactivity: global action for public health. Lancet, v. 380, n. 9838, p. 294-305, Jul 21 2012. ISSN 0140-6736.

KRUGER, J.; LANKFORD, T.; SCHMID, T. State legislative support for parks. Environ Health Insights, v. 4, p. 27-31, 2010.

KRUGER, J.; MOWEN, A. J.; LIBRETT, J. Recreation, parks, and the public health agenda: developing collaborative surveillance frameworks to measure leisure time activity and active park use. J Phys Act Health, v. 4 Suppl 1, p. S14-23, 2007.

LEE, A. C.; JORDAN, H. C.; HORSLEY, J. Value of urban green spaces in promoting healthy living and wellbeing: prospects for planning. Risk Manag Healthc Policy, v. 8, p. 131-7, 2015.

LIBRETT, J. et al. An introduction to parks, recreation, and public health: collaborative frameworks for promoting physical activity. J Phys Act Health, v. 4, supl 1, p. S1-13, 2007.

LIBRETT, J. J.; YORE, M. M.; SCHMID, T. L. Local ordinances that promote physical activity: a survey of municipal policies. Am J Public Health, v. 93, n. 9, p. 1399-403, Sep 2003. MCCRACKEN, D. S.; ALLEN, D. A.; GOW, A. J. Associations between urban greenspace and health-related quality of life in children. Prev Med Rep, v. 3, p. 211-21, Jun 2016. 
MENDES, R. et al. Promoção da saúde e redes de lideranças. Physis Revista de Saúde Coletiva, v. 23, n. 1, p. 209-226, 2013.

OLIVEIRA, S. M.; SANTOS, M. P.; SILVA, P. Crianças nos parques: segurança acima de tudo. Physis Revista de Saúde Coletiva, v. 28, n. 2, p. 23, 2018.

PEARCE, M. et al. Who children spend time with after school: associations with objectively recorded indoor and outdoor physical activity. Int J Behav Nutr Phys Act, v. 11, n. 1, p. 45, Mar 302014.

ROSS, S. E.; FRANCIS, L. A. Physical activity perceptions, context, barriers, and facilitators from a Hispanic child's perspective. Int J Qual Stud Health Well-being, v. 11, p. 31949, 2016.

SALLIS, J. F. et al. An ecological approach to creating active living communities. Annu Rev Public Health, v. 27, p. 297-322, 2006.

SALLIS, J. F. et al. Role of built environments in physical activity, obesity, and cardiovascular disease. Circulation, v. 125, n. 5, p. 729-737, 2012.

SANTOS, M. P. et al. Parental physical activity, safety perceptions and children's independent mobility. BMC Public Health, v. 13, p. 584, Jun 152013.

SPANGLER, K. J.; CALDWELL, L. L. The implications of public policy related to parks, recreation, and public health: a focus on physical activity. Journal of physical activity \& health, v. 4, supl 1, p. S64-71, 2007.

TESTER, J. M. The built environment: designing communities to promote physical activity in children. Pediatrics, v. 123, n. 6, p. 1591-8, Jun 2009.

VIJAY, G. C. et al. Are brief interventions to increase physical activity cost-effective? A systematic review. Br J Sports Med., v. 50, n. 7, Oct 52015.

WARD, J. S. et al. The impact of children's exposure to greenspace on physical activity, cognitive development, emotional wellbeing, and ability to appraise risk. Health Place, v. 40, p. 44-50, Jul 2016.

WORLD HEALTH ORGANIZATION. Global recommendations on physical activity for health. Geneva: WHO, 2010.

\section{Nota}

${ }^{1}$ S. M. de Oliveira: concepção e delineamento do estudo, coleta, tratamento, análise e interpretaçáo dos resultados, redaçáo e revisão crítica do conteúdo intelectual. M. P. M. dos Santos: concepção e delineamento do estudo, revisão crítica do conteúdo intelectual. P. Silva: análise e interpretação dos resultados, revisão crítica do conteúdo intelectual 


\section{Abstract}

\section{Local public policies to promote children's}

physical activity in public parks

Evidence reveals the potential of public parks to promote physical activity and health. The valorization of these environments for the promotion of children's physical activity can be an asset in public policies for health promotion and prevention of children's sedentary lifestyle. This study aimed to analyze public policies to promote children's physical activity in public parks in the municipality of Guimarães, Portugal. Individual semi-structured interviews were conducted with local public political managers. Data processing was carried out according to the qualitative methodology of content analysis, from which emerged the following themes related to the promotion of children's physical activity: political partnership (public and intersectoral), strategies and local constraints. The results revealed the need for greater public efforts to enhance public parks to promote children's physical activity. Promoting local public policies that favor local intersectoral partnership may be an effective strategy for promoting physical activity for children in public parks.

Keywords: public policy; recreational parks; child; physical activity. 